\title{
Evaluation of Impregnated Diamond Bit based on Image Processing Technology
}

\author{
Chong Wang ${ }^{1}$, Linhong $\mathrm{Xu}^{2, *}$ and Jinfeng $\mathrm{Zhen}^{2}$ \\ ${ }^{1}$ School of Information and Communication Engineering, University of Electronic Science and Technology of China, Chengdu, \\ 611731, China \\ ${ }^{2}$ Division of Mechanical engineering and Electronic Information, China University of Geosciences (Wuhan), Wuhan 430074, \\ China \\ ${ }^{*}$ Corresponding author
}

\begin{abstract}
Impregnated diamond bit are widely used in very difficult formation drilling. In the drilling process, the diamond particles embedded in the impregnated diamond bit will inevitable wear, loss and adhesive wear. In this case, the bonding between diamond particles and matrix and the diamond performance play a very important role for the impregnated diamond bit life and drilling efficiency. This study analyzed and processed the collected diamond pictures by using image processing and numerical calculation to obtain the information on the dislodge and protrude of diamond particle and then get some conclusion on the retention capability of matrix on particle.
\end{abstract}

Keywords-impregnated diamond bit; image processing; retention capability

\section{INTRODUCTION}

As a kind of bit used in very difficult formation drilling, Impregnated diamond bit are widely used in geological drilling, engineering geological exploration and rock and soil drilling excavation construction tool, in geological drilling, engineering geological exploration and other fields [1]. Usually, Impregnated diamond bit are composed of diamond particles embedded into a metal matrix and the main role of the metal matrix is to carry and retain diamonds particles for as long as possible.

While drilling, the rigid diamond particles pass over the mineral constituents and at same time itself matrix will be abraded and eroded to expose fresh diamond crystals which will take over the cutting action from those mechanically degraded and dislodged. The mechanically degrade and dislodged rate and amount of diamond particles will directly determine the drilling performance [2-4]. A well-selected matrix must hold diamond particles firmly and also guarantee an adequate wear adjusted to diamond lose. So, the retention capability between diamond particles and matrix play a very important role for the impregnated diamond bit life and drilling efficiency. Many researchers pay much more attention on the diamond retention capacity of the matrix from the many different sides. Some focus on the improve formula of matrix to get better retention [5-7], some use software to analyze different matrix materials influence on diamond retention [810]. Some focus on the diamond retention, wear mechanism and wear behavior by using scanning electron microscopy (SEM) micrographs, and SEM micrographs analysis concentrate much more on one or two diamond particle performance instead of from Macro-view of a whole drill bit [11-12]. Due to the constraints of the research methods, an effective quantitative measurement and evaluation of diamond retention is still an issue difficult to be solved.

In view of all these limitations, this paper tried to use macro image of drill bit to estimate the diamond retention capacity of the matrix by counting the number of diamonds and pullouts on the tool working face and calculating the proportion of the total number of diamonds to the total number of diamonds and pullouts.

\section{Diamond Particle Pull-OUt AND Retention}

\section{A. Dislodged Ratio of the Diamond}

The pull-out (or dislodged) of the diamond particles is a process that a diamond bit must go through during drilling, which play a very important role on the drilling efficiency. During drilling, the matrix has suffered wear, and diamonds presented different protrusion height, which is extremely beneficial to the performance and tool life, where these higher protrusion height diamonds began to cleave, crack or flatten and then are detached (pull-out) from the bonding matrix. The dislodged ratio of the diamond reflects the dislodged amount of the diamond particles during drilling which can be calculated as a ratio between the numbers of dislodged and the total number of the diamond particles distributed on the sample region which chosen randomly on the lip surface of the diamond drill bit. To a certain extent, the dislodged ratio of diamond can be seen as one index reflecting retention between the bit matrix and the diamond particles. To ensure the reliability of data, an average value should be used to by calculating from the data of several random sample regions.

\section{B. Pit's Area Left by the Diamond Particle Detached}

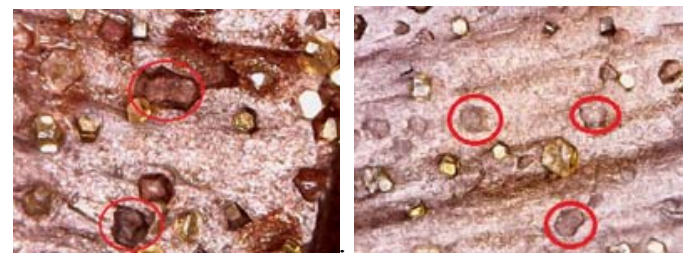

FIGURE I. DISLODGED TYPES OF DIAMOND PARTICLE. 
The cross-sectional area of the dislodged pit can give us some useful information about the situation on diamond pullout occurred. When the cross-sectional area of the dislodged pit is larger than or close to the maximum cross-sectional area of the diamond particles, it means that diamond particle pull-out but without reaching their half tool life, which regarded to be the premature dislodged. While, the cross-sectional area of the pull-out pit is smaller than the maximum cross-sectional area of the diamond particles, which means that the diamond particle pull-out when it reach at least over half tool life, which regarded as the second dislodged type. So, the cross-sectional area of the dislodged pit can provide us information on dislodged type and dislodged situation and also reflect the retention between matrix and diamond particle.

\section{Diamond Particle Employed in Impregnated Diamond Bit}

In order to analyze conveniently, here we take diamond particle as a sphere model approximately. Impregnated diamond bits studied in this paper is a kind of drill bit used in relatively hard strata drilling. Diamond mesh is chosen as 35/40 mesh, with corresponding diameters $500 / 425 \mu \mathrm{m}$, as shown in Fig.2.

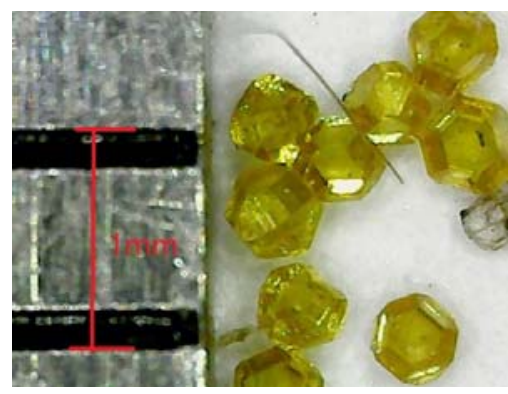

FIGURE II. DIAMOND PARTICLRE

\section{METHODS}

\section{A. Image Acquisition}

In this paper, the electronic magnifier was adopted as the image acquisition device for its advantage of low cost, convenient carrying and use, particularly suitable for image acquisition in the field or on the production site, although its imaging quality is not as good as other sophisticated imaging tools such as the scanning electron microscopy and the digital microscope. Electronic magnifier can get magnification 10 to 600 times image of the object (shown in Fig.3).

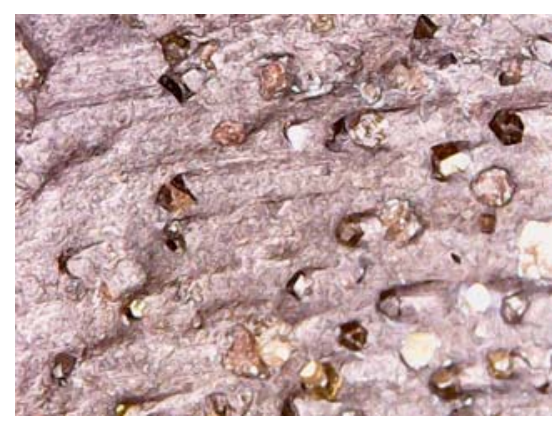

FIGURE III. PICUTURE OBSERVED.

\section{B. Image Preprocessing}

Usually, the image obtained from the electronic magnifier should be preprocessed to eliminate or reduce image quality issues such as uneven image brightness, low image contrast and high image noise, which may bring a larger error for the following-up image quantitative analysis and calculation. Among these issues, uneven image brightness can be eliminated by subtracting the estimated uneven brightness of the background from the original image. And the low image contrast can be improved by adjusting the gray-level histogram of images. High image noise can be deal with noise reduction processing. These processing will improve the image quality greatly and the image features such as diamond particles and the dislodged pit can be reinforced for the following-up analysis. Through a series preprocessing procedure, a series of image can be obtained shown as in Fig.4, from which, the feature of diamond particle and dislodged pit can be extracted and indentified clearly.

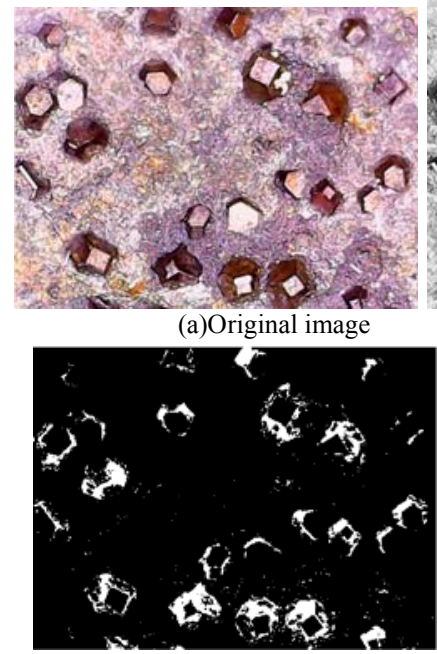

(e)Binary images
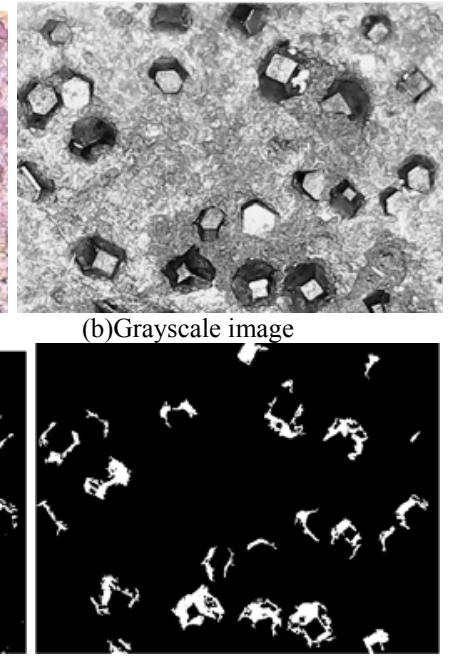

(f)Removal small noise points
FIGURE IV. IMAGE PREPROCESSING.

\section{Image Segmentation}

Usually, in the traditional pregnant diamond drill bit, the location of each diamond particle involved cannot be controlled and randomly present in the matrix. In this case, mutual adhesion together (shown in Fig.5) of some diamond particles will inevitably appear, and these adhesion area will result the identification and calculation error. Normally, classical watershed segmentation algorithm is a useful tool for image segmentation which can produce a continuous boundary to obtain an enclosed area. Here, marker-controlled watershed algorithm is used to solve mutual blocking adhesion area of two or three diamond particles. The results after segmentation process is shown in Fig 5, from which two diamond particles mutual adhesion areas can be segmented very well. The segmentation results obtained by watershed segmentation algorithm are shown in the Fig.5 


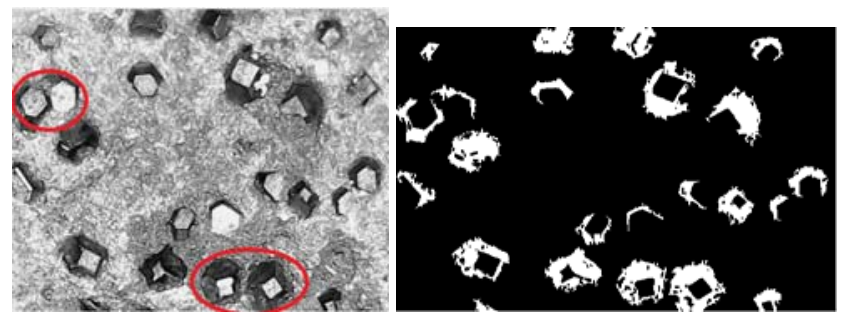

FIGURE V. SEGMENTED RESULT

\section{Sampling of the Diamond Drill Bits}

In this paper, seven impregnated diamond bits from different suppliers for the same formation drilling which means the size of the diamond particles are basically same were used to analyze, and the working life and drill effect of these seven were documented to analyze and compare with one another.

\section{SOFTWARE OF THE DiAmond DRILl Bit DEVELOPMENT}

\section{A. Function Design}

To analyze and calculate different situations of the diamond particles conveniently and automatically, here, analysis software of the diamond drill bit is developed based on the interface of MATLAB GUI. This software (shown in Fig.6) can perform following functions:

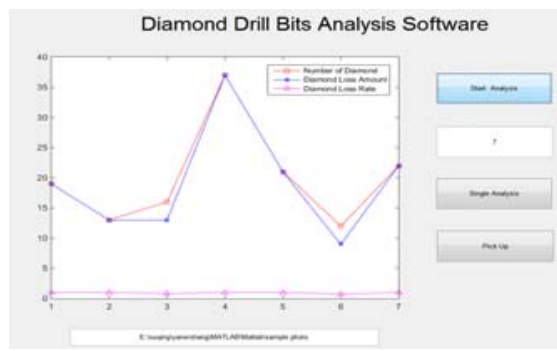

FIGURE VI. DIAMOND DRILL BITS ANALSIS SOFTWARE.

(1) Distribution and diamond particles dislodged situation analysis on a specific sampling area from one picture: the number of the diamond particles and the dislodged ratio of the diamond particles, as well as the area of the dislodged pits of the diamond particles contained within one microscopic image of the pits are quantitative analyzed;

(2) Comparative analysis of a group of picture from the different drill bits: Comparative analysis of a group of picture from the different drill bits can be made and present the result in line-chart form. A group of images can be analyzed and the results can be shown in the form of curves. The inspection of each parameter in this group of pictures can be compared and shown in a more intuitive way through the curve. Meanwhile, the results can be stored into the corresponding Excel spreadsheet to facilitate the detailed analysis in the future.

(3) Measurement of the specific dislodged area chosen: the dislodged pits of the diamond particles can be selected manually, the area and diameter of its equivalent circle are measured, namely, the cross sectional area and diameter of the diamond particles.
This software can recognize and calculate not only calculate the number of diamond particles and the number of diamond particles pull-out and a corresponding diamond particles pullout ratio of a specific sampling area automatically, but also can measure the specific pull-off area chosen manually by using the equivalent circle area of a pull-off pit.

\section{B. Measurement of the Cross-Sectional Area of the Specific Dislodged Pit}

The dislodged amount and dislodged ratio of the diamond particles can only illustrate the dislodged situation but without providing us any more information on the dislodged type as well as wear situation and probably reason caused dislodged. So, cross-sectional area of the dislodged pit was used. Here, the ROIPOLY function of the MATLAB digital image processing was employed to measure the cross-sectional area of pull-out pit chosen, which allow users to select polygon area interactively and set the corner of the polygonal area and finally obtain a masking binary image. And software will detect the binary image automatically which then be equivalent to the same size of the circular area, shown in Fig.7. Furthermore, Measurement results of dislodged pit area will be compared with the maximum cross-sectional area of the diamond particles in the same magnification to explain the dislodged situation of the diamond particles (shown in Fig.7).

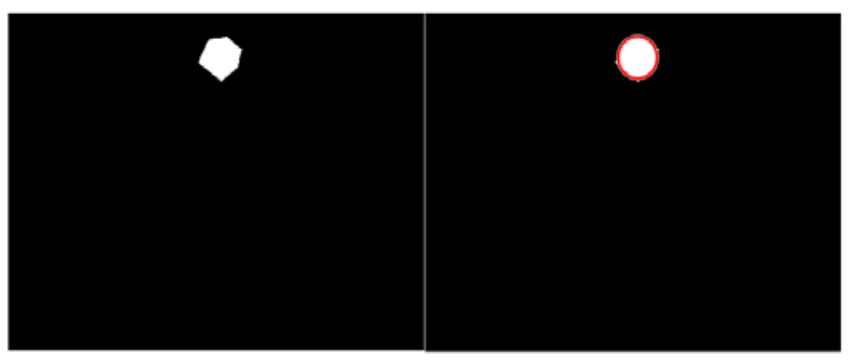

FIGURE VII. MEASUREMENT OF CROSS-SECTIONAL AREA.

\section{RESUltS AND DisCUSSIONS}

\section{A. Analysis of the Distribution and Dislodged Situations of the Diamond Particles}

As shown in Fig.8, the quantitative analysis results can be drawn in a way of line graph through software processing. The line chart about the information within the sampling area pictures of above 6 different bits including the total amount of diamond particles (pull-out and unpull-out), amount of dislodged diamond particles as well as dislodged ratio of the diamond are shown in Fig.8. As shown in the Fig.8, for the number of the diamond particles within the sampling area, the maximum is $2 \#$ drill bit while minimum is $6 \#$ drill bit, for the dislodged amount within the sampling area, the maximum is $2 \#$ drill bit while minimum is $6 \#$ drill bit, for the dislodged ratio of the diamond particles, the maximum is $3 \#$ drill bit while the minimum dislodged ratio is $2 \#$. However, the dislodged amount can not explain the quality of the drill since that the total number of the diamond particles itself examined is not the same. 


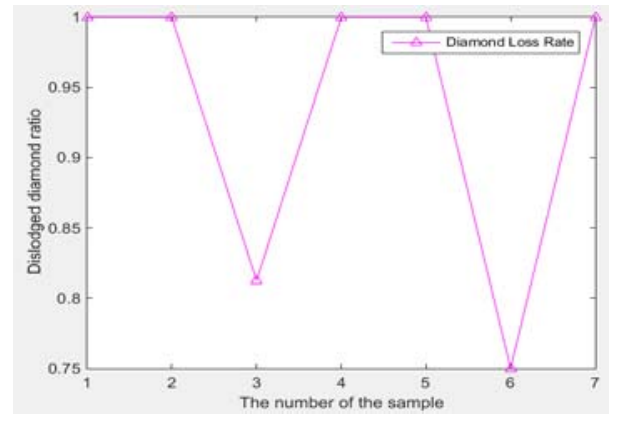

FIGURE VIII. DISLODGED DIAMOND PARTICLE RATIO

\section{B. Cross-Sectional Area}

In this paper, six drill bits are sampled and measured. Here, for comparison conveniently, pixel area was used as the unit of area of dislodged pit and the pixel area of the maximum crosssectional area of the diamond particles mentioned above (shown in Fig.2) is 1590.6. For 2\# drill bit, the dislodged pits cross-sectional areas of are almost all smaller than the maximum cross-sectional area of the diamond particles. While, the ratio of the cross-sectional areas of dislodged pits larger than the maximum cross-sectional area of the diamond particles of the $1 \#, 3 \#, 4 \#, 5 \#, 6 \#, 7 \#$ drill bit is $50 \%, 22.2 \%, 73.3 \%$, $64.7 \%, 84.6 \%$ and $64.7 \%$, and among of which, the $6 \#$ drill bit is particularly evident.

From comprehensive comparison such as the dislodged amount, dislodged ratio and the cross-sectional area of the dislodged pits of the diamond within the sampling areas, the best performance of this above group bits should be $1 \#$.

\section{CONCLUSION}

Under practical working conditions, the actual life of each drill is 5 meters for $1 \#$ drill bit, and 4 meters for $2 \#, 1.5$ meters for $3 \#, 4.3$ meters for $4 \#, 3.7$ meters for $5 \#, 2.9$ meters for $6 \#$ and 3.9 meters for $7 \#$. We can find that the $1 \#$ drill bit is with longest lifetime and the $3 \#$ drill bit shortest, while others lifetime is between them.

Then back again on the diamond amount of lip surface, the dislodged amount and the dislodged ratio of the diamond particles of the $1 \#$ drill bit, we can find that all these data of $1 \#$ drill bit are among intermediate value. At same time, for the 1\# drill bit, the dislodged pits areas larger than the maximum cross-sectional area of the diamond particles accounts for $50 \%$, among of them, most of value is close to the maximum crosssectional area of the diamond particles, which means most of the diamond particles detached until it is wore more than half.

This can be explained although the matrix of $1 \#$ is a little bit soft, but still the drilling effect is good enough and its performance can satisfy the basic needs of the strata. Thus, the lifetime and drilling effect is a result of the combination factor. Among this group of drill bits, the worst one may be $3 \#$. We can find that the diamond amount of the lip surface is nearly the same with $1 \#$, but the dislodged amount and dislodged ratio of the diamond particles both exceed $1 \#$ and the difference between them is relatively evident. And for $3 \#$ drill bit, although there is not too many the diamond whose areas of the dislodged pits bigger than the maximum cross-sectional area of the diamond particles, the area close to the maximum crosssectional area is still numerous. This situation illustrate that most of the diamond particles have been detached before wore half and the little remaining diamond particles are used to cut the rock. The situation results in the bad efficiency and the rapid wear of the drill. So, the life of $3 \#$ is shortest and the performance of $3 \#$ is relatively poor.

In summary, a diamond bit analysis software was designed based on Matlab image processing tool which can deal with pictures automatically. And at same time, a batch of drill bits from practical engineering field was chosen for image processing and analyzing. The result of analysis showed that the evaluation index proposed and the method for detecting the evaluation index can partly reflect the performance and the situation of drill bit and the matrix of the bit.

\section{REFERENCES}

[1] Songcheng Tan, Xiaohong Fang, Kaihua Yang, Longchen Duan. A new composite impregnated diamond bit for extra-hard, compact, and nonabrasive rock formation, Int. Journal of Refractory Metals and Hard Materials, 43 (2014) 186-192.

[2] W. Polini $\cdot$ S. Turchetta. Evaluation of diamond tool wear. International Journal Advanced Manufacture Technology (2005) 26: 959-964.

[3] Steven W. Webb. Diamond retention in sintered cobalt bonds for stone cutting and drilling. Diamond and Related Materials, 8 (1999) 20432052.

[4] S.Y. LUO. Characterisitics of diamond sawblade wear in sawing. Int. J. Mach.ToolsManufact.Vol.36, No, 6, pp. 661-672, 1996.

[5] S.Y. Luo. Effect of fillers of resin-bonded composites on diamond retention and wear behavior, Wear 2361999 339-349.

[6] Xipeng Xu, Xiaorui Tie, Hairong Wu.The effects of a Ti coating on the performance of metal-bonded diamond composites containing rare earth, International Journal of Refractory Metals \& Hard Materials, 25 (2007) 244-249.

[7] Luciano Jose de Oliveira, Guerold Sergueevitch Bobrovnitchii, Marcello Filgueira. Processing and characterization of impregnated diamond cutting tools using a ferrous metal matrix, International Journal of Refractory Metals \& Hard Materials 25 (2007) 328-335.

[8] J. KONSTANTY, A. ROMANSKI. NUMERICAL ANALYSIS OF DIAMOND RETENTION IN COBALT AND A COPPER-BASE ALLOY, A R C H I V E S O F M E T A L L U R G Y A N D M A T E R I A L S, Volume 592014 Issue 4 DOI: 10.2478/amm-2014-0247.

[9] J. Konstanty, Diamond bonding and matrix wear mechanisms involved in circular sawing of stone, Ind. Diamond Rev. 60, 55-65 (2000).

[10] Luciano Jose ' de Oliveira, Guerold Sergueevitch Bobrovnitchii, Marcello Filgueira. Processing and characterization of impregnated diamond cutting tools using a ferrous metal matrix, International Journal of Refractory Metals \& Hard Materials 25 (2007) 328-335.

[11] B. L i, P.M. A m a r a l, L. R e is, C.A. A n j i n h o, L.G. R o s a, M. d e $\mathrm{F} \mathrm{r}$ e i t a s, 3-D-modelling of the local plastic deformation and residual stresses of PM diamond-metal matrix composites, Computational Materials Science 47, 1023-1030 (2010).

[12] Xipeng Xu, Xiaorui Tie, Hairong Wu. The eVects of a Ti coating on the performance of metal-bonded diamond composites containing rare earth, International Journal of Refractory Metals \& Hard Materials 25 (2007) 244-249. 